TAMKANG JOURNAL OF MATHEMATICS

Volume 33, Number 4, Winter 2002

\title{
ON SOME INEQUALITIES RELATED TO OPIAL-TYPE INEQUALITY IN TWO VARIABLES
}

\author{
GOU-SHENG YANG AND TIEN-SHOU HUANG
}

\begin{abstract}
In this paper, we generalized some inequalities related to Opial-Type inequality in two variables. The analysis used in the proofs is quite elementary.
\end{abstract}

\section{Introduction}

In 1982, G. S. Yang [1] proved the following Opial-Type inequality in two variables:

Theorem A. If $f(s, t), f_{1}(s, t)$, and $f_{12}(s, t)$ are continuous functions on $[a, b] \times[c, d]$, and if $f(a, t)=f(b, t)=f_{1}(s, c)=f_{1}(s, d)=0$, for $a \leq s \leq b, c \leq t \leq d$, then

$$
\int_{a}^{b} \int_{c}^{d}|f(s, t)|\left|f_{12}(s, t)\right| d t d s \leq \frac{(b-a)(d-c)}{8} \int_{a}^{b} \int_{c}^{d}\left|f_{12}(s, t)\right|^{2} d t d s
$$

In 1983, C. T. Lin and G. S. Yang [2] generalized (1) in the following form:

Theorem B. If $f(s, t), f_{1}(s, t)$, and $f_{12}(s, t)$ are continuous functions on $[a, b] \times[c, d]$, and if $f(a, t)=f(b, t)=f_{1}(s, c)=f_{1}(s, d)=0$, for $a \leq s \leq b, c \leq t \leq d$, then

$$
\int_{a}^{b} \int_{c}^{d}|f(s, t)|^{m}\left|f_{12}(s, t)\right|^{n} d t d s \leq\left(\frac{n}{m+n}\right)\left[\frac{(b-a)(d-c)}{4}\right]^{m} \int_{a}^{b} \int_{c}^{d}\left|f_{12}(s, t)\right|^{m+n} d t d s
$$

In 1984 and 1986, B. G. Pachpatte [3,4] generalized (1) in the following forms:

Theorem C. Let $f(s, t), f_{1}(s, t), f_{12}(s, t)$ and $g(s, t), g_{1}(s, t), g_{12}(s, t)$ be continuous functions on $[a, b] \times[c, d]$, and let $f(a, t)=f(b, t)=f_{1}(s, c)=f_{1}(s, d)=0, g(a, t)=$ $g(b, t)=g_{1}(s, c)=g_{1}(s, d)=0$, for $a \leq s \leq b, c \leq t \leq d$. Then

$$
\begin{aligned}
& \int_{a}^{b} \int_{c}^{d}|f(s, t) g(s, t)|^{m}\left[|f(s, t)|\left|g_{12}(s, t)\right|+|g(s, t)|\left|f_{12}(s, t)\right|\right] d t d s \\
\leq & \frac{1}{2(m+1)}\left[\frac{(b-a)(d-c)}{4}\right]^{2 m+1} \int_{a}^{b} \int_{c}^{d}\left[\left|f_{12}(s, t)\right|^{2(m+1)}+\left|g_{12}(s, t)\right|^{2(m+1)}\right] d t d s
\end{aligned}
$$

Received March 11, 2002.

2000 Mathematics Subject Classification. 26D15.

Key words and phrases. Integral inequalities, Opial-Type inequality, Holder inequality, convex functions. 
Theorem D. Let $f(s, t), f_{1}(s, t), f_{12}(s, t)$ be real-valued continuous functions on $[a, b] \times[c, d]$, and $f(a, t)=f(b, t)=f_{1}(s, c)=f_{1}(s, d)=0$, for $a \leq s \leq b, c \leq t \leq d$. If $H$ is a convex increasing function for $x>0$ and $H(0)=0$, then

$$
\begin{aligned}
\int_{a}^{b} \int_{c}^{d} H^{\prime}(|f(s, t)|)\left|f_{12}(s, t)\right| d t d s \leq & H\left(\int_{a}^{\alpha} \int_{c}^{\beta}\left|f_{12}(s, t)\right| d t d s\right)+H\left(\int_{a}^{\alpha} \int_{\beta}^{d}\left|f_{12}(s, t)\right| d t d s\right) \\
& +H\left(\int_{\alpha}^{b} \int_{c}^{\beta}\left|f_{12}(s, t)\right| d t d s\right)+H\left(\int_{\alpha}^{b} \int_{\beta}^{d}\left|f_{12}(s, t)\right| d t d s\right)
\end{aligned}
$$

for $a \leq \alpha \leq b, c \leq \beta \leq d$.

The aim of this paper is to establish some new integral inequalities which cover the inequalities (2), (3) and (4).

\section{Main Results}

Throughout, we assume that $n$ is a real number such that $n \geq 1$, and $k=\left[\frac{(b-a)(d-c)}{4}\right]^{n-1}$.

Theorem 1. For $i=1,2$, let $f_{i}(s, t), D_{1} f_{i}(s, t), D_{2} D_{1} f_{i}(s, t)$ be real-valued continuous functions on $[a, b] \times[c, d]$, and $f_{i}(a, t)=f_{i}(b, t)=D_{1} f_{i}(s, c)=D_{1} f_{i}(s, d)=0$, for $a \leq s \leq b, c \leq t \leq d$. Let $H_{i}$ be convex, increasing functions on $[0, \infty)$ such that $H_{i}(0)=0$. Then

$$
\begin{aligned}
& \int_{a}^{b} \int_{c}^{d}\left[H_{1}\left(\left|f_{1}(s, t)\right|^{n}\right) H_{2}{ }^{\prime}\left(\left|f_{2}(s, t)\right|^{n}\right)\left|D_{2} D_{1} f_{2}(s, t)\right|^{n}\right. \\
& \left.+H_{2}\left(\left|f_{2}(s, t)\right|^{n}\right) H_{1}{ }^{\prime}\left(\left|f_{1}(s, t)\right|^{n}\right)\left|D_{2} D_{1} f_{1}(s, t)\right|^{n}\right] d t d s \\
\leq & \frac{1}{k_{1}} \prod_{i=1}^{2} H_{i}\left(k_{1} \int_{a}^{\alpha} \int_{c}^{\beta}\left|D_{2} D_{1} f_{i}(s, t)\right|^{n} d t d s\right)+\frac{1}{k_{2}} \prod_{i=1}^{2} H_{i}\left(k_{2} \int_{a}^{\alpha} \int_{\beta}^{d}\left|D_{2} D_{1} f_{i}(s, t)\right|^{n} d t d s\right) \\
+ & \frac{1}{k_{3}} \prod_{i=1}^{2} H_{i}\left(k_{3} \int_{\alpha}^{b} \int_{c}^{\beta}\left|D_{2} D_{1} f_{i}(s, t)\right|^{n} d t d s\right)+\frac{1}{k_{4}} \prod_{i=1}^{2} H_{i}\left(k_{4} \int_{\alpha}^{b} \int_{\beta}^{d}\left|D_{2} D_{1} f_{i}(s, t)\right|^{n} d t d s\right)
\end{aligned}
$$

where

$$
\begin{aligned}
& k_{1}=[(\alpha-a)(\beta-c)]^{n-1}, k_{2}=[(\alpha-a)(d-\beta)]^{n-1}, k_{3}=[(b-\alpha)(\beta-c)]^{n-1}, \\
& k_{4}=[(b-\alpha)(d-\beta)]^{n-1}, \text { and } a \leq \alpha \leq b, c \leq \beta \leq d .
\end{aligned}
$$

Proof. For $a \leq s \leq \alpha \leq b, c \leq t \leq \beta \leq d$, and $i=1,2$; define $Z_{i}(s, t)=$ $\int_{a}^{s} \int_{c}^{t}\left|D_{2} D_{1} f_{i}(u, v)\right|^{\bar{n}} d v d \bar{u}$. Then, $Z_{i}(s, t) \leq Z_{i}(s, \beta)^{\prime}, Z_{i}(a, t)=0$, and $D_{1} Z_{i}(s, t)=$ 
$\int_{c}^{t}\left|D_{2} D_{1} f_{i}(s, v)\right|^{n} d v$. Since $\left|f_{i}(s, t)\right|^{n} \leq\left(\int_{a}^{s}\left|D_{1} f_{i}(u, t)\right| d u\right)^{n}$ and $\left|D_{1} f_{i}(s, t)\right|^{n} \leq$ $\left(\int_{c}^{t}\left|D_{2} D_{1} f_{i}(s, v)\right| d v\right)^{n}$, it follows from Holder inequality with indices $\frac{n-1}{n}$ and $\frac{1}{n}$ that

$$
\left|f_{i}(s, t)\right|^{n} \leq(s-a)^{n-1}\left(\int_{a}^{s}\left|D_{1} f_{i}(u, t)\right|^{n} d u\right)
$$

and

$$
\left|D_{1} f_{i}(s, t)\right|^{n} \leq(t-c)^{n-1}\left(\int_{c}^{t}\left|D_{2} D_{1} f_{i}(s, v)\right|^{n} d v\right)=(t-c)^{n-1} D_{1} Z_{i}(s, t),
$$

so that $\left|f_{i}(s, t)\right|^{n} \leq k_{1} Z_{i}(s, \beta)$.

Since $H_{i}, H_{i}{ }^{\prime}$ are increasing on $[0, \infty), D_{2} D_{1} Z_{i}(s, t)=\left|D_{2} D_{1} f_{i}(s, t)\right|^{n}, D_{1} Z_{i}(s, c)=0$, and $H_{1}(0)=0$, we have

$$
\begin{aligned}
& \int_{a}^{\alpha} \int_{c}^{\beta}\left[H_{1}\left(\left|f_{1}(s, t)\right|^{n}\right) H_{2}{ }^{\prime}\left(\left|f_{2}(s, t)\right|^{n}\right)\left|D_{2} D_{1} f_{2}(s, t)\right|^{n}\right. \\
& \left.+H_{2}\left(\left|f_{2}(s, t)\right|^{n}\right) H_{1}{ }^{\prime}\left(\left|f_{1}(s, t)\right|^{n}\right)\left|D_{2} D_{1} f_{1}(s, t)\right|^{n}\right] d t d s \\
\leq & \int_{a}^{\alpha} \int_{c}^{\beta}\left[H_{1}\left(k_{1} Z_{1}(s, \beta)\right) H_{2}{ }^{\prime}\left(k_{1} Z_{2}(s, \beta)\right) D_{2} D_{1} Z_{2}(s, t)\right. \\
& \left.+H_{2}\left(k_{1} Z_{2}(s, \beta)\right) H_{1}{ }^{\prime}\left(k_{1} Z_{1}(s, \beta)\right) D_{2} D_{1} Z_{1}(s, t)\right] d t d s \\
= & \int_{a}^{\alpha}\left[H_{1}\left(k_{1} Z_{1}(s, \beta)\right) H_{2}{ }^{\prime}\left(k_{1} Z_{2}(s, \beta)\right) D_{1} Z_{2}(s, \beta)\right. \\
= & \frac{1}{k_{1}} \int_{a}^{\alpha} \frac{d}{d s}\left[H_{1}\left(k_{1}\left(k_{1} Z_{2}(s, \beta)\right) H_{1}{ }^{\prime}\left(k_{1} Z_{1}(s, \beta)\right) D_{1} Z_{1}(s, \beta)\right] d s\right. \\
= & \left.\frac{1}{k_{1}} \prod_{i=1}^{2} H_{i}\left(k_{1} Z_{2}(s, \beta)\right)\right] d s
\end{aligned}
$$

For $a \leq s \leq \alpha \leq b, c \leq \beta \leq t \leq d, i=1,2$, define $Z_{i}(s, t)=\int_{a}^{s} \int_{t}^{d}\left|D_{2} D_{1} f_{i}(u, v)\right|^{n} d v d u$ Then, $Z_{i}(s, t) \leq Z_{i}(\alpha, t), Z_{i}(a, t)=0$ and $D_{2} Z_{i}(s, t)=-\int_{a}^{s}\left|D_{2} D_{1} f_{i}(u, t)\right|^{n} d u$ Since $\left|f_{i}(s, t)\right|^{n} \leq\left(\int_{a}^{s}\left|D_{1} f_{i}(u, t)\right| d u\right)^{n}$ and $\left|D_{1} f_{i}(s, t)\right|^{n} \leq\left(\int_{t}^{d}\left|D_{2} D_{1} f_{i}(s, v)\right| d v\right)^{n}$, it follows from Holder inequality with indices $\frac{n-1}{n}$ and $\frac{1}{n}$ that

$$
\begin{aligned}
& \left|f_{i}(s, t)\right|^{n} \leq(s-a)^{n-1}\left(\int_{a}^{s}\left|D_{1} f_{i}(u, t)\right|^{n} d u\right) \\
& \left|D_{1} f_{i}(s, t)\right|^{n} \leq(d-t)^{n-1}\left(\int_{t}^{d}\left|D_{2} D_{1} f_{i}(s, v)\right|^{n} d v\right)=(d-t)^{n-1} D_{1} Z_{i}(s, t),
\end{aligned}
$$


so that

$$
\begin{aligned}
\left|f_{i}(s, t)\right|^{n} & \leq(s-a)^{n-1}(d-t)^{n-1}\left(\int_{a}^{s} D_{1} Z_{i}(u, t) d u\right) \\
& =(s-a)^{n-1}(d-t)^{n-1} Z_{i}(s, t) \\
& \leq k_{2} Z_{i}(\alpha, t)
\end{aligned}
$$

Since $H_{i}, H_{i}{ }^{\prime}$ are increasing on $[0, \infty), D_{2} D_{1} Z_{i}(s, t)=-\left|D_{2} D_{1} f_{i}(s, v)\right|^{n}, D_{2} Z_{i}(a, t)=0$, and $H_{1}(0)=0$, we have

$$
\begin{aligned}
& \int_{a}^{\alpha} \int_{\beta}^{d}\left[H_{1}\left(\left|f_{1}(s, t)\right|^{n}\right) H_{2}{ }^{\prime}\left(\left|f_{2}(s, t)\right|^{n}\right)\left|D_{2} D_{1} f_{2}(s, t)\right|^{n}\right. \\
& \left.+H_{2}\left(\left|f_{2}(s, t)\right|^{n}\right) H_{1}{ }^{\prime}\left(\left|f_{1}(s, t)\right|^{n}\right)\left|D_{2} D_{1} f_{1}(s, t)\right|^{n}\right] d t d s \\
\leq & -\int_{a}^{\alpha} \int_{\beta}^{d}\left[H_{1}\left(k_{2} Z_{1}(\alpha, t)\right) H_{2}{ }^{\prime}\left(k_{2} Z_{2}(\alpha, t)\right) D_{2} D_{1} Z_{2}(s, t)\right. \\
\left.\quad+H_{2}\left(k_{2} Z_{2}(\alpha, t)\right) H_{1}{ }^{\prime}\left(k_{2} Z_{1}(\alpha, t)\right) D_{2} D_{1} Z_{1}(s, t)\right] d t d s & -\int_{\beta}^{d}\left[H_{1}\left(k_{2} Z_{1}(\alpha, t)\right) H_{2}{ }^{\prime}\left(k_{2} Z_{2}(\alpha, t)\right) D_{2} Z_{2}(\alpha, t)\right. \\
\left.\quad+H_{2}\left(k_{2} Z_{2}(\alpha, t)\right) H_{1}{ }^{\prime}\left(k_{2} Z_{1}(\alpha, t)\right) D_{2} Z_{1}(\alpha, t)\right] d t & \frac{1}{k_{2}} \prod_{i=1}^{2} H_{i}\left(k_{2} \int_{a}^{\alpha} \int_{\beta}^{d}\left|D_{2} D_{1} f_{i}(u, v)\right|^{n} d v d u\right)
\end{aligned}
$$

Similarly, we have

$$
\begin{aligned}
& \int_{\alpha}^{b} \int_{c}^{\beta}\left[H _ { 1 } ( | f _ { 1 } ( s , t ) | ^ { n } ) H _ { 2 } { } ^ { \prime } \left(\left|f_{2}(s, t)\right|^{n}\left|D_{2} D_{1} f_{2}(s, t)\right|^{n}\right.\right. \\
& \left.+H_{2}\left(\left|f_{2}(s, t)\right|^{n}\right) H_{1}{ }^{\prime}\left(\left|f_{1}(s, t)\right|^{n}\right)\left|D_{2} D_{1} f_{1}(s, t)\right|^{n}\right] d t d s \\
\leq & \frac{1}{k_{3}} \prod_{i=1}^{2} H_{i}\left(k_{3} \int_{\alpha}^{b} \int_{c}^{\beta}\left|D_{2} D_{1} f_{i}(u, v)\right|^{n} d v d u\right),
\end{aligned}
$$

and

$$
\begin{aligned}
& \int_{\alpha}^{b} \int_{\beta}^{d}\left[H_{1}\left(\left|f_{1}(s, t)\right|^{n}\right) H_{2}{ }^{\prime}\left(\left|f_{2}(s, t)\right|^{n}\right)\left|D_{2} D_{1} f_{2}(s, t)\right|^{n}\right. \\
& \left.+H_{2}\left(\left|f_{2}(s, t)\right|^{n}\right) H_{1}{ }^{\prime}\left(\left|f_{1}(s, t)\right|^{n}\right)\left|D_{2} D_{1} f_{1}(s, t)\right|^{n}\right] d t d s \\
\leq & \frac{1}{k_{4}} \prod_{i=1}^{2} H_{i}\left(k_{4} \int_{\alpha}^{b} \int_{\beta}^{d}\left|D_{2} D_{1} f_{i}(u, v)\right|^{n} d v d u\right) .
\end{aligned}
$$

The desired inequality then follows from (6), (7), (8), and (9). 
Remark 1. Let $n=1, H_{1}=H, H_{2}=1, f_{1}=f$ in Theorem 1. Then, it follows from the inequality (5) that

$$
\begin{aligned}
\int_{a}^{b} \int_{c}^{d} H^{\prime}(|f(s, t)|)\left|f_{12}(s, t)\right| d t d s \leq & H\left(\int_{a}^{\alpha} \int_{c}^{\beta}\left|f_{12}(s, t)\right| d t d s\right)+H\left(\int_{a}^{\alpha} \int_{\beta}^{d}\left|f_{12}(s, t)\right| d t d s\right) \\
& +H\left(\int_{\alpha}^{b} \int_{c}^{\beta}\left|f_{12}(s, t)\right| d t d s\right)+H\left(\int_{\alpha}^{b} \int_{\beta}^{d}\left|f_{12}(s, t)\right| d t d s\right)
\end{aligned}
$$

which is the inequality (4) [see[4], Theorem 1]

Theorem 2. Let $f_{i}(s, t), D_{1} f_{i}(s, t), D_{2} D_{1} f_{i}(s, t), H_{i}$ be as in Theorem 1. Then

$$
\begin{aligned}
& \int_{a}^{b} \int_{c}^{d}\left[H_{1}\left(\left|f_{1}(s, t)\right|\right)^{n} H_{2}{ }^{\prime}\left(\left|f_{2}(s, t)\right|^{n}\right)\left|D_{2} D_{1} f_{2}(s, t)\right|^{n}\right. \\
& \left.+H_{2}\left(\left|f_{2}(s, t)\right|^{n}\right) H_{1}{ }^{\prime}\left(\left|f_{1}(s, t)\right|^{n}\right)\left|D_{2} D_{1} f_{1}(s, t)\right|^{n}\right] d t d s \\
\leq & \frac{1}{k}\left[\prod_{i=1}^{2} H_{i}\left(k \int_{a}^{\frac{a+b}{2}} \int_{c}^{\frac{c+d}{2}}\left|D_{2} D_{1} f_{i}(s, t)\right|^{n} d t d s\right)+\prod_{i=1}^{2} H_{i}\left(k \int_{a}^{\frac{a+b}{2}} \int_{\frac{c+d}{2}}^{d}\left|D_{2} D_{1} f_{i}(s, t)\right|^{n} d t d s\right)\right. \\
& \left.+\prod_{i=1}^{2} H_{i}\left(k \int_{\frac{a+b}{2}}^{b} \int_{c}^{\frac{c+d}{2}}\left|D_{2} D_{1} f_{i}(s, t)\right|^{n} d t d s\right)+\prod_{i=1}^{2} H_{i}\left(k \int_{\frac{a+b}{2}}^{b} \int_{\frac{c+d}{2}}^{d}\left|D_{2} D_{1} f_{i}(s, t)\right|^{n} d t d s\right)\right]
\end{aligned}
$$

Proof. By taking $\alpha=\frac{a+b}{2}, \beta=\frac{c+d}{2}$ in Theorem 1, we have $k_{1}=k_{2}=k_{3}=k_{4}=k$. Hence, the inequality (10) follows from (5).

Remark 2. Let $n=1, m \geq 0, f_{1}=f, f_{2}=g, H_{1}=H_{2}=x^{m+1}$ in Theorem 2. Then, it follows from the inequality (10) that

$$
\begin{aligned}
& (m+1) \int_{a}^{b} \int_{c}^{d}|f(s, t) g(s, t)|^{m}\left[|f(s, t)|\left|g_{12}(s, t)\right|+|g(s, t)|\left|f_{12}(s, t)\right|\right] d t d s \\
\leq & {\left[\left(\int_{a}^{\frac{a+b}{2}} \int_{c}^{\frac{c+d}{2}}\left|f_{12}(s, t)\right| d t d s\right)\left(\int_{a}^{\frac{a+b}{2}} \int_{c}^{\frac{c+d}{2}}\left|g_{12}(s, t)\right| d t d s\right)\right]^{m+1} } \\
+ & {\left[\left(\int_{a}^{\frac{a+b}{2}} \int_{\frac{c+d}{2}}^{d}\left|f_{12}(s, t)\right| d t d s\right)\left(\int_{a}^{\frac{a+b}{2}} \int_{\frac{c+d}{2}}^{d}\left|g_{12}(s, t)\right| d t d s\right)\right]^{m+1} } \\
+ & {\left[\left(\int_{\frac{a+b}{2}}^{b} \int_{c}^{\frac{c+d}{2}}\left|f_{12}(s, t)\right| d t d s\right)\left(\int_{\frac{a+b}{2}}^{b} \int_{c}^{\frac{c+d}{2}}\left|g_{12}(s, t)\right| d t d s\right)\right]^{m+1} } \\
+ & {\left[\left(\int_{\frac{a+b}{2}}^{b} \int_{\frac{c+d}{2}}^{d}\left|f_{12}(s, t)\right| d t d s\right)\left(\int_{\frac{a+b}{2}}^{b} \int_{\frac{c+d}{2}}^{d}\left|g_{12}(s, t)\right| d t d s\right)\right]^{m+1} }
\end{aligned}
$$


Using Holder inequality twice with indices $\frac{2 m+1}{2(m+1)}$ and $\frac{1}{2(m+1)}$, we have

$$
\begin{aligned}
& {\left[\int_{a}^{\frac{a+b}{2}} \int_{c}^{\frac{c+d}{2}}\left|f_{12}(s, t)\right| d t d s\right]^{m+1} } \\
\leq & {\left[\int_{a}^{\frac{a+b}{2}}\left(\frac{d-c}{2}\right)^{\frac{2 m+1}{2(m+1)}}\left(\int_{c}^{\frac{c+d}{2}}\left|f_{12}(s, t)\right|^{2(m+1)} d t\right)^{\frac{1}{2(m+1)}} d s\right]^{m+1} } \\
\leq & \left(\frac{d-c}{2}\right)^{\frac{2 m+1}{2}}\left(\frac{b-a}{2}\right)^{\frac{2 m+1}{2}}\left(\int_{a}^{\frac{a+b}{2}} \int_{c}^{\frac{c+d}{2}}\left|f_{12}(s, t)\right|^{2(m+1)} d t d s\right)^{\frac{1}{2}}
\end{aligned}
$$

Also,

$$
\begin{aligned}
& {\left[\int_{a}^{\frac{a+b}{2}} \int_{c}^{\frac{c+d}{2}}\left|g_{12}(s, t)\right| d t d s\right]^{m+1} } \\
\leq & \left(\frac{d-c}{2}\right)^{\frac{2 m+1}{2}}\left(\frac{b-a}{2}\right)^{\frac{2 m+1}{2}}\left(\int_{a}^{\frac{a+b}{2}} \int_{c}^{\frac{c+d}{2}}\left|g_{12}(s, t)\right|^{2(m+1)} d t d s\right)^{\frac{1}{2}}
\end{aligned}
$$

Since $2 x y \leq x^{2}+y^{2}$ for all $x$ and $y$, we have

$$
\begin{aligned}
& {\left[\left(\int_{a}^{\frac{a+b}{2}} \int_{c}^{\frac{c+d}{2}}\left|f_{12}(s, t)\right| d t d s\right)\left(\int_{a}^{\frac{a+b}{2}} \int_{c}^{\frac{c+d}{2}}\left|g_{12}(s, t)\right| d t d s\right)\right]^{m+1} } \\
\leq & \frac{1}{2}\left[\frac{(b-a)(d-c)}{4}\right]^{2 m+1} \int_{a}^{\frac{a+b}{2}} \int_{c}^{\frac{c+d}{2}}\left(\left|f_{12}(s, t)\right|^{2(m+1)}+\left|g_{12}(s, t)\right|^{2(m+1)}\right) d t d s .
\end{aligned}
$$

Similarly,

$$
\begin{aligned}
& {\left[\left(\int_{a}^{\frac{a+b}{2}} \int_{\frac{c+d}{2}}^{d}\left|f_{12}(s, t)\right| d t d s\right)\left(\int_{a}^{\frac{a+b}{2}} \int_{\frac{c+d}{2}}^{d}\left|g_{12}(s, t)\right| d t d s\right)\right]^{m+1} } \\
\leq & \frac{1}{2}\left[\frac{(b-a)(d-c)}{4}\right]^{2 m+1} \int_{a}^{\frac{a+b}{2}} \int_{\frac{c+d}{2}}^{d}\left(\left|f_{12}(s, t)\right|^{2(m+1)}+\left|g_{12}(s, t)\right|^{2(m+1)}\right) d t d s, \\
& {\left[\left(\int_{\frac{a+b}{2}}^{b} \int_{c}^{\frac{c+d}{2}}\left|f_{12}(s, t)\right| d t d s\right)\left(\int_{\frac{a+b}{2}}^{b} \int_{c}^{\frac{c+d}{2}}\left|g_{12}(s, t)\right| d t d s\right)\right]^{m+1} } \\
\leq & \frac{1}{2}\left[\frac{(b-a)(d-c)}{4}\right]^{2 m+1} \int_{\frac{a+b}{2}}^{b} \int_{c}^{\frac{c+d}{2}}\left(\left|f_{12}(s, t)\right|^{2(m+1)}+\left|g_{12}(s, t)\right|^{2(m+1)}\right) d t d s,
\end{aligned}
$$

and

$$
\begin{aligned}
& {\left[\left(\int_{\frac{a+b}{2}}^{b} \int_{\frac{c+d}{2}}^{d}\left|f_{12}(s, t)\right| d t d s\right)\left(\int_{\frac{a+b}{2}}^{b} \int_{\frac{c+d}{2}}^{d}\left|g_{12}(s, t)\right| d t d s\right)\right]^{m+1} } \\
\leq & \frac{1}{2}\left[\frac{(b-a)(d-c)}{4}\right]^{2 m+1} \int_{\frac{a+b}{2}}^{b} \int_{\frac{c+d}{2}}^{d}\left(\left|f_{12}(s, t)\right|^{2(m+1)}+\left|g_{12}(s, t)\right|^{2(m+1)}\right) d t d s .
\end{aligned}
$$


Hence,

$$
\begin{aligned}
& \int_{a}^{b} \int_{c}^{d}|f(s, t) g(s, t)|^{m}\left[|f(s, t)|\left|g_{12}(s, t)\right|+|g(s, t)|\left|f_{12}(s, t)\right|\right] d t d s \\
\leq & \frac{1}{2(m+1)}\left[\frac{(b-a)(d-c)}{4}\right]^{2 m+1} \int_{a}^{b} \int_{c}^{d}\left[\left|f_{12}(s, t)\right|^{2(m+1)}+\left|g_{12}(s, t)\right|^{2(m+1)}\right] d t d s
\end{aligned}
$$

which is the inequality (3) [See [3], Theorem 1]

Remark 3. Let $H_{1}=x^{\frac{m+n}{n}}, H_{2}=1, f_{1}=f_{2}=f$ in Theorem 2. Then for $m \geq 0$, $n \geq 1$, it follows from the inequality (10) that

$$
\begin{aligned}
& \int_{a}^{b} \int_{c}^{d}\left(\frac{m+n}{n}\right)|f(s, t)|^{m}\left|f_{12}(s, t)\right|^{n} d t d s \\
\leq & \frac{1}{k}\left[\left(k \int_{a}^{\frac{a+b}{2}} \int_{c}^{\frac{c+d}{2}}\left|f_{12}(s, t)\right|^{n} d t d s\right)^{\frac{m+n}{n}}+\left(k \int_{a}^{\frac{a+b}{2}} \int_{\frac{c+d}{2}}^{d}\left|f_{12}(s, t)\right|^{n} d t d s\right)^{\frac{m+n}{n}}\right. \\
& \left.+\left(k \int_{\frac{a+b}{2}}^{b} \int_{c}^{\frac{c+d}{2}}\left|f_{12}(s, t)\right|^{n} d t d s\right)^{\frac{m+n}{n}}+\left(k \int_{\frac{a+b}{2}}^{b} \int_{\frac{c+d}{2}}^{d}\left|f_{12}(s, t)\right|^{n} d t d s\right)^{\frac{m+n}{n}}\right]
\end{aligned}
$$

Using Holder inequality twice with indices $\frac{m}{m+n}$ and $\frac{n}{m+n}$ to each term on the right hand side, we have

$$
\begin{aligned}
& \left(\int_{a}^{\frac{a+b}{2}} \int_{c}^{\frac{c+d}{2}}\left|f_{12}(s, t)\right|^{n} d t d s\right)^{\frac{m+n}{n}} \leq\left[\frac{(b-a)(d-c)}{4}\right]^{\frac{m}{n}}\left(\int_{a}^{\frac{a+b}{2}} \int_{c}^{\frac{c+d}{2}}\left|f_{12}(s, t)\right|^{m+n} d t d s\right), \\
& \left(\int_{a}^{\frac{a+b}{2}} \int_{\frac{c+d}{2}}^{d}\left|f_{12}(s, t)\right|^{n} d t d s\right)^{\frac{m+n}{n}} \leq\left[\frac{(b-a)(d-c)}{4}\right]^{\frac{m}{n}}\left(\int_{a}^{\frac{a+b}{2}} \int_{\frac{c+d}{2}}^{d}\left|f_{12}(s, t)\right|^{m+n} d t d s\right), \\
& \left(\int_{\frac{a+b}{2}}^{b} \int_{c}^{\frac{c+d}{2}}\left|f_{12}(s, t)\right|^{n} d t d s\right)^{\frac{m+n}{n}} \leq\left[\frac{(b-a)(d-c)}{4}\right]^{\frac{m}{n}}\left(\int_{\frac{a+b}{2}}^{b} \int_{c}^{\frac{c+d}{2}}\left|f_{12}(s, t)\right|^{m+n} d t d s\right),
\end{aligned}
$$

and

$$
\left(\int_{\frac{a+b}{2}}^{b} \int_{\frac{c+d}{2}}^{d}\left|f_{12}(s, t)\right|^{n} d t d s\right)^{\frac{m+n}{n}} \leq\left[\frac{(b-a)(d-c)}{4}\right]^{\frac{m}{n}}\left(\int_{\frac{a+b}{2}}^{b} \int_{\frac{c+d}{2}}^{d}\left|f_{12}(s, t)\right|^{m+n} d t d s\right) .
$$

Therefore,

$$
\int_{a}^{b} \int_{c}^{d}|f(s, t)|^{m}\left|f_{12}(s, t)\right|^{n} d t d s \leq\left(\frac{n}{m+n}\right)\left[\frac{(b-a)(d-c)}{4}\right]^{m} \int_{a}^{b} \int_{c}^{d}\left|f_{12}(s, t)\right|^{m+n} d t d s
$$

which is the inequality (2) [see [2], Theorem 5] 


\section{References}

[1] G. S. Yang, Inequality of Opial-Type in two variables, Tamkang J. Math. 13 (1982), 255-259.

[2] C. T. Lin and G. S. Yang, A generaized Opial's inequality in two variables, Tamkang J. Math. 15 (1983), 115-122.

[3] B. G. Pachpatte, On Opial-Type inequalities in two variables, Proc. Royal Soc. Edinburgh, 100A(1985), 263-270.

[4] B. G. Pachpatte, On Yang type integral inequalities, Tamkang J. Math. 18 (1987), 89-96.

Department of Mathematics, Tamkang University, Tamsui, Taiwan 25137.

Department of Information Management, Kaohsiung University of Applied Sciences, Kaohsiung, Taiwan 807. 